\title{
Current Options for the Diagnosis, Staging and Therapeutic Management of Colorectal Cancer
}

\author{
Goro Nakayama Chie Tanaka Yasuhiro Kodera \\ Department of Gastroenterological Surgery (Surgery II), Nagoya University Graduate \\ School of Medicine, Nagoya, Japan
}

\section{Key Message}

The management of colorectal cancer (CRC) should be undertaken using a multi-modal approach, taking into account the extent, localization and biology of the tumor, as well as individual patient factors.

\section{Practical Implications}

The initial diagnosis of CRC is generally done using colonoscopy, accompanied by histological confirmation of the biopsy. Important staging factors are the depth of tumor invasion, regional lymph node (LN) involvement and distant metastases. The standard curative treatment for colon cancer is surgical resection including colectomy with optimal $\mathrm{LN}$ dissection. For rectal cancer, an accurate diagnosis of tumor location, T stage, $\mathrm{N}$ stage and relationship of the tumor to the mesorectal fascia are important for defining a therapeutic strategy. Surgery, however, is also the mainstay of treatment. For patients with stage III colon cancer, the benefits of adjuvant therapy using the FOLFOX regimen are well-established. However, this has not been formally demonstrated for rectal cancer patients. For those with resectable liver metastases, the use of perioperative FOLFOX4 regimen resulted in improved 3-year disease-free survival, though the optimal sequence of chemotherapy still remains to be defined. Finally, the management of metastatic CRC involves the use of various drugs used either alone or in combination, including 5-FU/ LV, capecitabine, irinotecan, oxaliplatin, bevacizumab, cetuximab, panitumumab, zivaflibercept and regorafenib. 
Nakayama et al.: Current Options for the Diagnosis, Staging and Therapeutic Management of Colorectal Cancer

\title{
Key Words
}

Colorectal cancer $\cdot$ Imaging modality $\cdot$ Therapeutic management

\begin{abstract}
Background: Colorectal cancer (CRC) is the one of the most common malignancies and is a leading cause of cancer death worldwide. The incidence of mortality from CRC has decreased in Western countries because of earlier diagnosis and improved treatment modalities; however, mortality is still increasing in most other regions of the world. Summary: Progress in the development of imaging modalities has enabled more accurate staging based on the TNM classification. The therapeutic management of CRC should involve a multi-modal approach, including high-quality surgery and an optimal choice of chemotherapy and radiotherapy regimens according to disease characteristics and patient preferences. Even in the case of metastatic disease, the optimal multi-modal treatments could achieve potential cure or long-term survival benefit in some patients. The aim of this review is to present current options regarding the diagnosis, staging and treatment management for CRC based on published research reports and the current management guidelines.

(C) 2013 S. Karger AG, Base
\end{abstract}

\section{Introduction}

Colorectal cancer (CRC) is one of the most common cancers; it is the first and fourth most frequently diagnosed cancer in Europe and the United States, respectively [1, 2]. Worldwide in $2008,1.23$ million cases of CRC were reported to be responsible for $9.7 \%$ of the total cancer burden, after lung (1.61 million cases) and breast cancer (1.38 million cases) [3]. Furthermore, CRC is one of the leading causes of cancer death worldwide; it is the second most common cause of cancer death in Europe and the United States [1-3]. Approximately 20-25\% of patients with CRC present with metastatic disease at the time of diagnosis, and $20-25 \%$ of patients will develop metastases after treatment, resulting in a relatively high overall mortality rate of $40-45 \%$ [2]. The treatment and outcome of early and advanced disease has steadily improved. During the past two decades the incidence of mortality from CRC has decreased in Europe and the United States, most likely because of earlier diagnosis through screening and improved treatment modalities [3]. However, mortality in most of the Asia-Pacific region is still increasing, particularly because of progressive westernization of lifestyle [4].

\section{Diagnosis and Staging}

An initial diagnosis of CRC is generally made using colonoscopy, with biopsy and histological confirmation of the diagnosis. If carcinoma is diagnosed, computed tomography (CT) of the chest, abdomen and pelvis is recommended for initial preoperative evaluation, staging and optimal therapeutic planning.

CRC is generally staged using the American Joint Commission on Cancer (AJCC) TNM system [5]. The Japanese Classification of Colorectal Carcinoma (JCCRC) (published by the Japanese Society for Cancer of the Colon and Rectum) is widely used for staging in Japan [6]. Important staging factors in both systems are tumor depth of invasion, regional lymph node (LN) involvement and distant metastasis. These classifications are clearly correlated with survival outcomes.

Preoperative staging of colon cancer should focus on the detection of local regional LNs and distant metastasis. This is because the standard treatment is surgical resection without 
preceding treatments, regardless of the information regarding $\mathrm{T}$ stage. CT can accurately assess the presence of distant metastatic disease, even though the overall accuracy of CT varies with the stage of colon cancer. The accuracy of CT has been reported to be $67 \%$ for $\mathrm{T}$ staging, $69 \%$ for $\mathrm{N}$ staging and $95 \%$ for $\mathrm{M}$ staging [7]. Other modalities for the evaluation of the M stage including magnetic resonance imaging (MRI; gadoxetic acid [Primovist]-enhanced) is helpful in the further diagnosis of equivocal liver lesions detected using CT. Fluorodeoxyglucose $\left({ }^{18} \mathrm{~F}\right)$ positron emission tomography should not be used routinely for initial staging; it is more sensitive than CT in ruling out extra-hepatic metastases. CT colonography (virtual colonoscopy) is not a standard procedure. Consequently, it could be helpful in the detection of synchronous colon cancers or polyps if colonoscopic examination is incomplete [8].

In terms of rectal cancer, the accurate diagnosis of tumor location, $\mathrm{T}$ stage, $\mathrm{N}$ stage and the relationship of the tumor to the mesorectal fascia (potential circumferential resection margin involvement) is important in defining the treatment strategy. MRI is the recommended modality for initial staging and preoperative planning in rectal cancer, because it can accurately define tumor localization, with regard to the relationship of the tumor to the peritoneal reflection or the anal sphincter complex. MRI provides superior resolution regarding the mucosal layers of the rectum and has the ability to predict the $\mathrm{T}$ stage in relation to an anatomical structure, including the mesorectal fascia [9]. Some studies have demonstrated that the distance between the tumor and the mesorectal fascia measured using MRI can predict a negative circumferential resection margin on subsequent histopathology [10]. MRI or multidetector $\mathrm{CT}$ are usually performed for the diagnosis of $\mathrm{N}$ stage, but these modalities are not accurate enough to predict nodal metastases; the sensitivity and specificity in detecting nodal metastases in rectal cancer were 66 and 76\%, respectively, for MRI and 55 and 74\%, respectively, for CT [11].

\section{Therapeutic Management}

The management of CRC should be a multi-modal approach according to tumor localization, extent and biology, and patient factors. Although optimal surgical resection is still the mainstay of curative treatment, optimal multi-modal treatments could maintain long-term survival, quality of life and even cure in selected patients. Treatment and management decisions regarding CRC must be founded on evidence-based guidelines, including the National Comprehensive Cancer Network (NCCN) and the European Society for Medical Oncology (ESMO) guidelines [2,8]. These guidelines are intended to establish standard treatment strategies for CRC and to eliminate disparities among the various institutions in terms of treatment.

\section{Surgery}

With regard to colon cancer, surgical resection including colectomy with optimal LN dissection is the mainstay of curative treatment, at least for stages $\geq \mathrm{T} 2 \mathrm{~N} 0 \mathrm{M} 0$. According to the ESMO guidelines, resection should include a segment of colon that is at least $5 \mathrm{~cm}$ in length on either side of the tumor. In the JCCRC guidelines, the length of the colon segment is defined by the classification of the pericolic LNs based on the location of the tumor and feeding artery [6]. There is a positive relationship between the number of LNs resected and accurate staging, risk of recurrence and survival outcomes in stage II or III CRC [12]. The current evidencebased standard according to the NCCN guidelines is that $\geq 12$ LNs be removed and examined for tumor invasion in order to accurately identify stage II cancer [13].

Laparoscopic colectomy may be an option for patients whose tumors are not located in the rectum or transverse colon, as well as for patients without locally advanced acute bowel obstruction or perforation [13]. Only experienced surgeons skilled in open abdominal ex- 
ploratory surgery should perform laparoscopic colectomy; both closed and open procedures are therapeutically equivalent when performed by skilled surgeons in institutions where these procedures are frequently conducted [14].

Surgery is also the mainstay of treatment for rectal cancer. Total mesorectal excision is the standard surgical procedure recommended for all rectal cancers localized in the middle and lower third of the rectum. Lateral LNs are defined as those that are drained by the middle rectal artery or that are located along the internal iliac vessels. In Japan, lateral LN dissection has been performed for advanced lower rectal cancer $(\geq \mathrm{T} 3)$ to reduce the risk of local relapse, even though the significance of the survival benefit remains unclear. The NCCN guidelines recommend chemoradiotherapy for patients with $\geq \mathrm{T} 3$ tumors or with LN metastasis irrespective of the invasion depth of the primary tumor [13].

Management of the primary tumor in the setting of unresectable metastases is still controversial. Recent pooled analysis has suggested that resection of the primary tumor may provide some survival benefit in the setting of unresectable metastases [15]. Conversely, the National Surgical Adjuvant Breast and Bowel Project (NSABP) C-10 trial showed that patients with an asymptomatic primary lesion and unresectable metastases who received mFOLFOX6 plus bevacizumab, without upfront resection of the primary, experienced an acceptable morbidity with an overall survival time of 19.9 months [16]. At this time, given the balance of risks and benefits associated with surgery, palliative resection of the primary tumor should only be considered for patients with unequivocal symptoms, likely obstruction or bleeding [2].

\section{Adjuvant Chemotherapy}

The benefit of adjuvant therapy for patients with stage III colon cancer has been established. The Multicenter International Study of Oxaliplatin/5-Fluorouracil/Leucovorin in the Adjuvant Treatment of Colon Cancer (MOSAIC) trial found that FOLFOX4 was clearly superior to 5-FU/LV only in the case of patients with stage III colon cancer [17]. This study established the FOLFOX regimen as the standard adjuvant setting for patients with stage III colon cancer. The results of several studies that have evaluated the other oxaliplatin-based regimens, FLOX or CapeOX (capecitabine/oxaliplatin), mirrored the outcome of the MOSAIC trial; these regimens are options for patients who are unable to tolerate FOLFOX. Conversely, adding irinotecan to adjuvant 5-FU/LV was not found to be of additional benefit in these clinical trials. 5-FU or capecitabine are recommended alternative drugs for patients who cannot be administered oxaliplatin.

There is currently no evidence that molecularly targeted agents such as the vascular endothelial growth factor (VEGF) inhibitor bevacizumab or the epidermal growth factor receptor (EGFR) inhibitors cetuximab or panitumumab have a role in the adjuvant setting of CRC. In the NSABP C-08 trial, patients with stage II and III colon cancer were randomized to bevacizumab plus mFOLFOX6 or to mFOLFOX6 alone. At 15 months, patients receiving bevacizumab had significantly longer disease-free survival, but 3 years after treatment there was no significant difference relative to the disease-free survival of patients treated with mFOLFOX6 alone [18]. Similarly, the AVANT trial involving a comparative evaluation of FOLFOX4, bevacizumab plus FOLFOX4, and bevacizumab plus oxaliplatin/capecitabine in patients with high-risk stage II or stage IIII colon cancer showed no benefit as a result of the addition of bevacizumab.

In relation to stage II colon cancer, the absolute benefit of adjuvant therapy has not yet been defined. In several studies, adjuvant therapy has been reported to offer only a 2-4\% survival advantage over surgery alone. However, a subset of patients with high-risk factors for recurrence related to the primary tumor characteristics might benefit from adjuvant therapy. The American Society of Clinical Oncology (ASCO) continues to recommend that 
patients with stage II CRC should not routinely receive adjuvant therapy; this may be considered for specific high-risk patients with T4 lesions, perforation, inadequate LN sampling or poorly differentiated histology. Similarly, the ESMO suggests that stage II patients must be separated into high- and low-risk groups, according to the presence of at least one of the following tumor-related risk factors: $\mathrm{LN}$ sampling $<12$, poorly differentiated tumor, vascular or lymphatic or perineural invasion, pT4 stage or clinical presentation with intestinal occlusion or perforation [8]. The standard regimen for high-risk stage II patients is still equivocal because the benefit of the addition of oxaliplatin to 5 -FU is very small. In the MOSAIC trial, the addition of oxaliplatin to the chemotherapy regimens of high-risk stage II patients did not achieve a significant trend regarding improvement in disease-free survival as compared with 5-FU alone [17]. However, recent analyses of the results from the NSABP C05-C08 trial demonstrated a 2-3\% benefit in the 5-year overall survival rate regarding the addition of oxaliplatin to 5-FU-based adjuvant chemotherapy in stage II colon cancer [19].

In contrast to colon cancer, the value of adjuvant chemotherapy after surgery and preoperative radiotherapy in rectal cancer is limited and its efficacy has not formally been proven. Although the standard treatment regimen is still equivocal, patients with stage III and II rectal cancer are usually treated with postoperative chemotherapy involving 5-FU or capecitabine, with or without oxaliplatin, based on data from colon cancer studies $[2,8]$.

\section{Chemotherapy for Resectable Metastatic CRC}

In patients with resectable liver metastases, the administration of perioperative FOLFOX4 has been shown to deliver a 9.2\% improvement in the 3-year disease-free survival relative to resection of liver metastases alone [20]. In the ESMO guidelines, the administration of perioperative chemotherapy, and preoperative and postoperative FOLFOX over 3 months is recommended for initially R0-resectable metastatic disease [8]. Recently, the New EPOC study evaluated the value of perioperative chemotherapy with the addition of cetuximab for resectable liver metastases in patients with wild-type $K R A S$; a lower progression-free survival time (14.1 months in the cetuximab plus chemotherapy arm vs. 20.5 months in the chemotherapy arm, $p=0.030$ ) was found in the cetuximab arm, with no additional improvement in response rate, resectability and pathological response. Theoretically, preoperative chemotherapy will help remove micrometastatic disease; in addition, in planning postoperative chemotherapy it will help determine whether or not the tumor is responsive to the regimen. However, the optimal sequencing of chemotherapy for patients with resectable metastatic disease remains unclear.

\section{Chemotherapy for Unresectable Metastatic CRC}

The current management of metastatic CRC involves various active drugs, given either in combination or as single agents; these are $5-\mathrm{FU} / \mathrm{LV}$, capecitabine, irinotecan, oxaliplatin, bevacizumab, cetuximab, panitumumab, ziv-aflibercept and regorafenib [2]. The selection of treatments is based on considerations regarding the aim of the therapy, tumor biology, patient-related factors and drug-related factors.

In the NCCN guidelines, available chemotherapies in the first-line setting are: FOLFOX \pm bevacizumab, CapeOX \pm bevacizumab, FOLFOX \pm panitumumab (KRAS wild-type gene), FOLFIRI \pm bevacizumab, FOLFIRI \pm cetuximab or panitumumab (KRAS wild-type gene), 5-FU/ LV or capecitabine \pm bevacizumab, and FOLFOXIRI [2]. Bevacizumab can be combined with all FU and oxaliplatin or irinotecan combinations. The addition of bevacizumab to either the irinotecan-based or the oxaliplatin-based regimen has been reported to significantly improve progression-free and overall survival [21]. However, the impact of bevacizumab on tumor response and shrinkage (including 'early tumor shrinkage' and 'deepness of response') is unclear; therefore, a bevacizumab-based combination therapy might not be preferentially 
Nakayama et al.: Current Options for the Diagnosis, Staging and Therapeutic Management of Colorectal Cancer

used in patients requiring maximum tumor shrinkage. In the $K R A S$ wild-type tumor, cetuximab in combination with FOLFIRI and panitumumab with FOLFOX has been shown to increase the response rate, progression-free survival and overall survival, particularly in the case of liver-limited disease [22]. Chemotherapy combined with anti-EGFR agents, especially with cetuximab, should be carefully selected. Combinations of cetuximab and oxaliplatin (+ capecitabine) could be harmful to patients with the KRAS wild-type tumor. Recently, in the FIRE-3 study, a head-to-head comparison of cetuximab and bevacizumab in a first-line setting was carried out. Overall survival was reported to be significantly longer in patients treated with FOLFIRI plus cetuximab relative to FOLFIRI plus bevacizumab, even though no difference in overall response rate (primary endpoint) and progression-free survival between both arms was observed. Definite information regarding the comparative efficacy of bevacizumab or anti-EGFR in combination with chemotherapy will soon be available from the US Intergroup trial (CALGB/SWOG 80405). The new targeted drug, aflibercept, has achieved a significantly increased response rate, progression-free survival and overall survival in combination with FOLFIRI in a second-line setting, including previous bevacizumab failures (AFFIRM trial) [23]. Regorafenib is a dual targeted VEGFR2-TIE2 tyrosine kinase inhibitor, which has been reported to deliver a significant improvement in progression-free and overall survival in third-/last-line therapy as a single agent as compared with a placebo [24].

The optimal choice of the first-line treatment should be based on the clinical presentation and patterns of tumor biology, resectability of the disease, patient-related factors and drugrelated factors. In the ESMO guidelines, patients with metastatic CRC are classified into four clinical groups: group 0, R0-resectable liver or lung metastases; group 1, liver or lung metastases that are not R0-resectable but might become resectable after downsizing using induction chemotherapy; group 2, multiple metastases with rapid progression or tumor-related symptoms; and group 3, multiple metastases without major symptoms and risk of rapid deterioration. In group 0 , the aim of treatment is cure and the first choice of treatment remains surgical resection. In group 1 , the most active combination regimen which can induce the maximum possible tumor shrinkage should be selected up front. In group 2, the aims of treatment are to achieve rapid regression of metastases and at least disease control. Therefore, an upfront relatively active combination regimen should be selected according to the tolerance of the patients. In group 3, the aims of treatment are prevention of tumor progression with symptom disappearance and prolongation of life with minimal treatment burden. Consequently, treatment should be selected according to disease characteristics and patient preference with regard to toxicity and efficacy [8].

In the Treatment across Multiple Lines (TML) trial, patients with metastatic CRC who progressed after treatment with regimens containing bevacizumab in a first-line setting received second-line therapy consisting of a changed chemotherapy backbone, with or without bevacizumab. Overall survival time was significantly longer in patients continuing on bevacizumab (hazard ratio $=0.81 ; p=0.0062$ ) [25]. Based on this study, the continuation of bevacizumab after progression (the bevacizumab beyond initial progression [BBP] strategy) was one of the standard strategies used in a second-line setting [2]. However, the indication for the BBP strategy should be carefully selected because the TML trial excluded patients with rapidly progressing disease (progression-free survival $<3$ months) in first-line treatment or with a poor performance status.

\section{Predictive Markers for Chemotherapy}

Predictive markers for efficacy are important in the individualization of the optimal chemotherapy; they are also important in avoiding unnecessary treatment, toxicity and the financial costs associated with therapy. The presence of the KRAS (exon 2) mutation can be used to exclude patients from treatment with EGFR antibodies. Recently, other mutations in 
Nakayama et al.: Current Options for the Diagnosis, Staging and Therapeutic Management of Colorectal Cancer

$R A S, K R A S$ (exons 3 and 4) and the NRAS genes have been reported as predictive markers that can be used in the selection of patients who would further benefit from treatment with EGFR antibodies. However, no further molecular markers are relevant to the decision regarding treatment, and the use of bevacizumab in particular. The BRAF mutation could potentially be employed as a prognostic rather than as a predictive marker, but it is not ready for routine use yet.

\section{Conclusion}

Progress in the development of imaging modalities has enabled more accurate staging, especially in rectal cancer. An optimal multi-modal approach including high-quality surgery and an optimal choice of chemotherapy and radiotherapy regimens according to disease characteristics and patient factors could achieve potential cure or long-term survival benefit even in the case of metastatic disease. The application of targeted agents has shown much promise in the treatment of metastatic CRC. Predictive markers are important in the individualization of the optimal treatment. The future offers hope that patients will have individualized therapies based on their tumor genetics.

\section{Funding Sources}

No specific funding was provided.

\section{Disclosure Statement}

The authors have no conflicts of interest to declare.

\section{References}

1 Ferlay J, Parkin DM, Steliarova-Foucher E: Estimates of cancer incidence and mortality in Europe in 2008. Eur J Cancer 2010;46:765-781.

-2 Benson AB III, Bekaii-Saab T, Chan E, Chen YJ, Choti MA, Cooper HS, Engstrom PF, Enzinger PC, Fakih MG, Fenton MJ, Fuchs CS, Grem JL, Hunt S, Kamel A, Leong LA, Lin E, May KS, Mulcahy MF, Murphy K, Rohren E, Ryan DP, Saltz L, Sharma S, Shibata D, Skibber JM, Small W Jr, Sofocleous CT, Venook AP, Willett CG, Gregory KM, Freedman-Cass DA: Metastatic colon cancer, version 3. 2013: featured updates to the NCCN Guidelines. J Natl Compr Canc Netw 2013;11:141-152.

-3 Ferlay J, Shin HR, Bray F, Forman D, Mathers C, Parkin DM: Estimates of worldwide burden of cancer in 2008: GLOBOCAN 2008. Int J Cancer 2010;127:2893-2917.

4 Ku G, Tan IB, Yau T, Boku N, Laohavinij S, Cheng AL, Kang YK, de Lima Lopes G Jr: Management of colon cancer: resource-stratified guidelines from the Asian Oncology Summit 2012. Lancet Oncol 2012;13:e470-e481.

5 Hari DM, Leung AM, Lee JH, Sim MS, Vuong B, Chiu CG, Bilchik AJ: AJCC Cancer Staging Manual 7th edition criteria for colon cancer: do the complex modifications improve prognostic assessment? J Am Coll Surg 2013; 217:181-190.

6 Watanabe T, Itabashi M, Shimada Y, Tanaka S, Ito Y, Ajioka Y, Hamaguchi T, Hyodo I, Igarashi M, Ishida H, Ishiguro M, Kanemitsu Y, Kokudo N, Muro K, Ochiai A, Oguchi M, Ohkura Y, Saito Y, Sakai Y, Ueno H, Yoshino T, Fujimori T, Koinuma N, Morita T, Nishimura G, Sakata Y, Takahashi K, Takiuchi H, Tsuruta O, Yamaguchi T, Yoshida M, Yamaguchi N, Kotake K, Sugihara K; Japanese Society for Cancer of the Colon and Rectum: Japanese Society for Cancer of the Colon and Rectum (JSCCR) guidelines 2010 for the treatment of colorectal cancer. Int J Clin Oncol 2012;7:1-29.

7 Leufkens AM, Van Den Bosch MA, Van Leeuwen MS, Siersema PD: Diagnostic accuracy of computed tomography for colon cancer staging: a systematic review. Scand J Gastroenterol 2011;46:887-894. 
Nakayama et al.: Current Options for the Diagnosis, Staging and Therapeutic Management of Colorectal Cancer

-8 Schmoll HJ, Van Cutsem E, Stein A, Valentini V, Glimelius B, Haustermans K, Nordlinger B, van de Velde CJ Balmana J, Regula J, Nagtegaal ID, Beets-Tan RG, Arnold D, Ciardiello F, Hoff P, Kerr D, Köhne CH, Labianca R, Price T, Scheithauer W, Sobrero A, Tabernero J, Aderka D, Barroso S, Bodoky G, Douillard JY, El Ghazaly H, Gallardo J, Garin A, Glynne-Jones R, Jordan K, Meshcheryakov A, Papamichail D, Pfeiffer P, Souglakos I, Turhal S, Cervantes A: ESMO Consensus Guidelines for management of patients with colon and rectal cancer. A personalized approach to clinical decision making. Ann Oncol 2012;23:2479-2516.

-9 Kaur H, Choi H, You YN, Rauch GM, Jensen CT, Hou P, Chang GJ, Skibber JM, Ernst RD: MR imaging for preoperative evaluation of primary rectal cancer: practical considerations. Radiographics 2012;32:389-409.

10 Glimelius B, Beets-Tan R, Blomqvist L, Brown G, Nagtegaal I, Påhlman L, Quirke P, Valentini V, van de Velde C: Mesorectal fascia instead of circumferential resection margin in preoperative staging of rectal cancer. J Clin Oncol 2011;29:2142-2143.

11 Bipat S, Glas AS, Slors FJ, Zwinderman AH, Bossuyt PM, Stoker J: Rectal cancer: local staging and assessment of lymph node involvement with endoluminal US, CT, and MR imaging - a meta-analysis. Radiology 2004;232: 773-783.

12 Berger AC, Sigurdson ER, LeVoyer T, Hanlon A, Mayer RJ, Macdonald JS, Catalano PJ, Haller DG: Colon cancer survival is associated with decreasing ratio of metastatic to examined lymph nodes. J Clin Oncol 2005;23: 8706-8712.

13 National Comprehensive Cancer Network Clinical Practice Guidelines: Colon cancer v.3.2012. Available at: http://www.nccn.org/professionals/physician_gls/PDF/colon.pdf.

14 Clinical Outcomes of Surgical Therapy Study Group: A comparison of laparoscopically assisted and open colectomy for colon cancer. N Engl J Med 2004;240:2050-2059.

15 Venderbosch S, de Wilt JH, Teerenstra S, Loosveld OJ, van Bochove A, Sinnige HA, Creemers GJ, Tesselaar ME, Mol L, Punt CJ, Koopman M: Prognostic value of resection of primary tumor in patients with stage IV colorectal cancer: retrospective analysis of two randomized studies and a review of the literature. Ann Surg Oncol 2011; 18:3252-3260.

16 McCahill LE, Yothers G, Sharif S, Petrelli NJ, Lai LL, Bechar N, Giguere JK, Dakhil SR, Fehrenbacher L, Lopa SH, Wagman LD, O'Connell MJ, Wolmark N: Primary mFOLFOX6 plus bevacizumab without resection of the primary tumor for patients presenting with surgically unresectable metastatic colon cancer and an intact asymptomatic colon cancer: definitive analysis of NSABP trial C-10. J Clin Oncol 2012;30:3223-3228.

-17 Andre T, Boni C, Mounedji-Boudiaf L, Navarro M, Tabernero J, Hickish T, Topham C, Zaninelli M, Clingan P, Bridgewater J, Tabah-Fisch I, de Gramont A; Multicenter International Study of Oxaliplatin/5-Fluorouracil/ Leucovorin in the Adjuvant Treatment of Colon Cancer (MOSAIC) Investigators: Oxaliplatin, fluorouracil, and leucovorin as adjuvant treatment for colon cancer. N Engl J Med 2004;350:2343-2351.

18 Allegra CJ, Yothers G, O’Connell MJ, Sharif S, Petrelli NJ, Colangelo LH, Atkins JN, Seay TE, Fehrenbacher L, Goldberg RM, O’Reilly S, Chu L, Azar CA, Lopa S, Wolmark N: Phase III trial assessing bevacizumab in stages II and III carcinoma of the colon: results of the NSABP protocol C-08. J Clin Oncol 2011;29:11-16.

19 Yothers G, Allegra C: The efficacy of oxaliplatin (Ox) when added to 5-fluorouracil/leucovorin (FU/L) in stage II colon cancer. J Clin Oncol 2011;29:abstr 3507.

20 Nordlinger B, Sorbye H, Collette L, Glimelius B, Poston GJ, Schlag PM, Rougier R, Bechstein W, Walpole E, Gruenberger T: Final results of the EORTC intergroup randomized phase III study 40983 [EPOC] evaluating the benefit of peri-operative FOLFOX4 chemotherapy for patients with potentially resectable colorectal cancer liver metastases. ASCO Annual Meeting Proceedings. J Clin Oncol 2007; 25:2s.

-21 Saltz LB, Clarke S, Diaz-Rubio E, Scheithauer W, Figer A, Wong R, Koski S, Lichinitser M, Yang TS, Rivera F, Couture F, Sirzén F, Cassidy J: Bevacizumab in combination with oxaliplatin-based chemotherapy as first-line therapy in metastatic colorectal cancer: a randomized phase III study. J Clin Oncol 2008;26:2013-2019.

-22 Van Cutsem E, Kohne CH, Lang I, Folprecht G, Nowacki MP, Cascinu S, Shchepotin I, Maurel J, Cunningham D, Tejpar S, Schlichting M, Zubel A, Celik I, Rougier P, Ciardiello F: Cetuximab plus irinotecan, fluorouracil, and leucovorin as first-line treatment for metastatic colorectal cancer: updated analysis of overall survival according to tumor KRAS and BRAF mutation status. J Clin Oncol 2011;29:2011-2019.

23 Pericay C, Folprecht G: Phase 2 randomized, noncomparative open-label study of aflibercept and modified FOLFOX6 in the first line treatment of metastatic colorectal cancer (AFFIRM). Ann Oncol 2012;23(suppl 4): abstr 0-0024.

24 Grothey A, Sobrero A: Results of a phase III randomized, double-blind, placebo controlled, multicenter trial (CORRECT) of regorafenib plus best supportive care (BSC) versus placebo plus BSC in patients (pts) with metastatic colorectal cancer (mCRC) who have progressed after standard therapies. J Clin Oncol 2012;30:abstr LBA 385.

25 Arnold D, Andre T, Bennouna J, et al: Bevacizumab (BEV) plus chemotherapy (CT) continued beyond first progression in patients with metastatic colorectal cancer (mCRC) previously treated with BEV plus CT: results of a randomized phase III intergroup study (TML study). J Clin Oncol 2012;30:abstr CRA3503. 